\title{
Early Clinical Outcomes with a 3-D Porous Titanium Acetabular Cup
}

\author{
Tatsuya Sueyoshi, E. Michael Keating, Merrill A. Ritter, John B. Meding, \\ Matthew J. Brunsman \\ Center for Hip and Knee Surgery, Joint Replacement Surgeons of Indiana Research Foundation (JRSI), \\ Mooresville, IN, USA \\ Email: jrsiresearch1@gmail.com
}

Received 23 February 2016; accepted 28 May 2016; published 31 May 2016

Copyright (C) 2016 by authors and Scientific Research Publishing Inc.

This work is licensed under the Creative Commons Attribution International License (CC BY).

http://creativecommons.org/licenses/by/4.0/

(c) (i) Open Access

\begin{abstract}
Cementless acetabular components are associated with a significant incidence of polyethylene wear and secondary osteolysis. 3-dimentional (3-D) porous coating and enhanced shell to a liner fixation are expected to reduce the polyethylene wear and to increase the longevity of an acetabular cup. The authors report the early clinical outcome with a cementless acetabular cup, Regenerex Ringloc+ (Zimmer Biomet, Warsaw, IN) for total hip arthroplasty. Between 2007 and 2013, the registry with $476 \mathrm{hip}$ joints was reviewed retrospectively. There were 210 (48.4\%) female and $224(51.6 \%)$ male patients. The average age of the patients at the surgery was $63.9( \pm 12.0)$ years and the average follow-up period was 2.6 years $[0.5,6.5]$. At the final follow-up, there was no loosening or cup failure. There were 2 infections, 1 dislocation and 1 liner fracture, resulting in revision hip surgery. Average Harris hip score improved from 53.9 preoperatively to 91.4 postoperatively. The survivorship of Regenerex Ring Loc+ cup and the low complication rate is comparable with previous studies using other 3-D porous metal prostheses. Although this short-term outcome from the institute is very encouraging, a longer follow-up study is required.
\end{abstract}

\section{Keywords}

Total Hip Arthroplasty (THA), 3-D Porous, Regenerex, Ringloc+, Clinical Outcome, Complication

\section{Introduction}

Cementless acetabular cup in total hip arthroplasty (THA) has evolved during the past few decades [1]. Many modern cementless acetabular cup designs and materials have shown excellent rates of bone ingrowth and longevity [2] [3]. Despite this evolution, acetabular cup loosening has been reported as the primary reason for revision 
THA [4]. This loosening usually occurs as a result of implant fixation failure and manifests as a motion between the prosthesis and bone. To enhance the biological fixation of these implants, most cementless acetabular components have added various porous coatings like those manufactured by sintering cobalt-chrome or titanium beads and the use of diffusion bonding of titanium fiber wires [5]. Pelvic osteolysis seen around cementless cups was attributed to the polyethylene wear debris resulting from micro motion between the nonarticulating side of the polyethylene liner and the interior of the metallic shell (backside wear). The Regenerex Ring Loc+ (Zimmer Biomet, Warsaw, IN) is an acetabular cup with a 3-dimentional (3-D) porous coating and an enhanced fixation of shell to a liner.

Regenerex refers to its 3-D porous coating and has been reported to show a high primary stability due to the scratch-fit (Figure 1). It also has a secondary stability due to osteogenesis and bone ingrowth into the porous titanium [6]. Ring Loc+ is an improved shell to liner connection system with a spring-loaded ring clamp that was previously adopted for the Ring Loc (Zimmer Biomet, Warsaw, IN) acetabular shell with more than 15 years (Figure 2) [7]. This prosthesis is sometimes used for revision hip surgery due to its highly stable fixation to an acetabulum with various augmentations [8], but the authors mainly used this for primary hip surgery expecting better bone ingrowth and longer survivorship. There are only two reports in the literature regarding the clinical

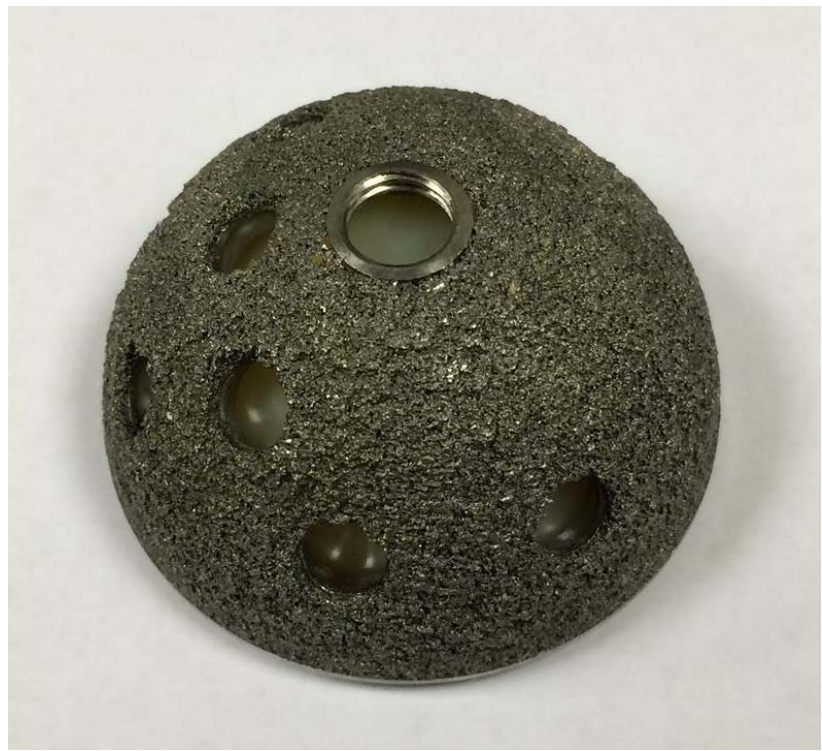

Figure 1. Rough porous surface of Regenerex Ring Loc+ cup with multiple screw holes.

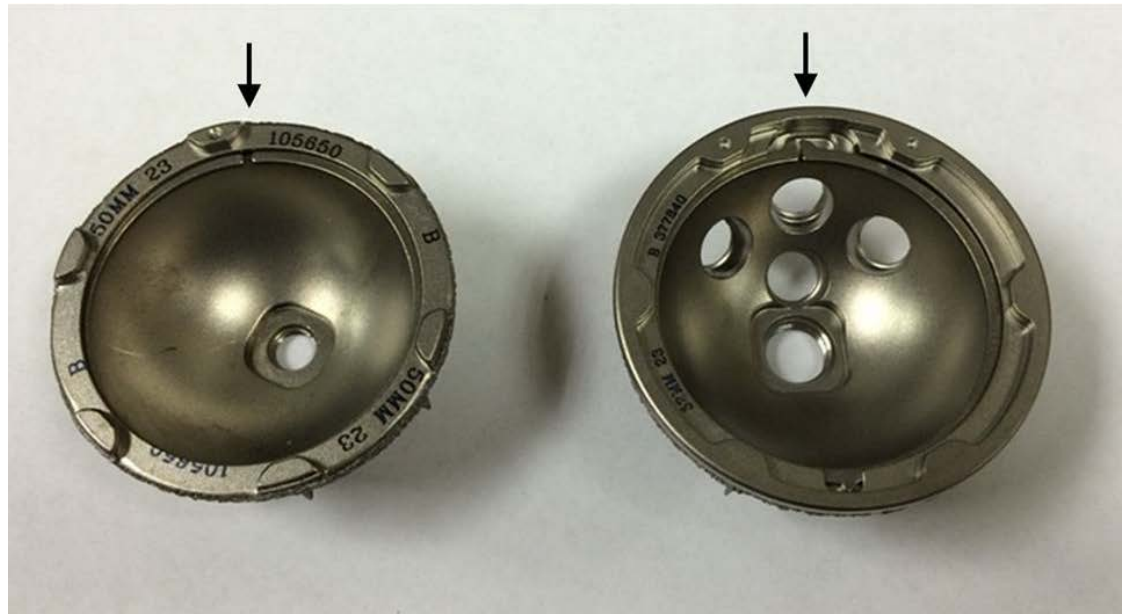

Figure 2. Left: Ring Loc cup, Right: Regenerex Ring Loc+ cup with limited screw holes. Arrows depict the change of locking rings. 
outcome with this prosthesis type [8] [9]. This study reports the early clinical outcome following cementless 3-D porus metal cup in total hip arthroplasty from a single institute.

\section{Patients and Methods}

The registry in the institution was reviewed retrospectively, 476 primary cementless THAs with the Regenerex Ring Loc+ acetabular cup between 2007 and 2013. The average follow-up time was 2.6 years [range: 0.5, 6.5]. Indications for THA were primary osteoarthritis in 435 hips, rheumatoid arthritis in 4 hips, osteonecrosis in 9 hips, femoral neck fracture 5 hips and others in 25 hips. There were 210 (48.4\%) female and 224 (51.6\%) male patients. The average age of the patients at the surgery was 63.9 ( \pm 12.0$)$ years [range: 30, 92]. All the cases were performed by board certificated experienced surgeons in the author's institute. Prior institutional review board approval was obtained by the IRB of this institution.

The Regenerex Ring Loc + shell is a porous titanium construct (average porosity of 67\%) available in 10 sizes (from 54 to $72 \mathrm{~mm}$ in $2 \mathrm{~mm}$ increments), each with multiple, limited screw holes or solid hemisphere without holes. The authors used all types of cups with or without screws. This prosthesis is designed to offer the option to stack augments in the most complex cases but the authors didn't use any augments.

Clinical evaluation was based on Harris hip score from the individual function scores and pain scores. All patients returned for follow-up at approximately 6 months, 1 and 3 years postoperatively. At each follow-up, patients were examined and assessed for surgical complications, including prolonged wound drainage, hematoma formation, superficial or deep infection.

\section{Results}

At the final follow-up, 357 out of 476 hips were followed as scheduled. The minimum follow-up time was 6 months and the maximum was 6.5 years. The average follow-up time was 2.6 years. There was no Regenerex cup failure and the survivorship with aseptic loosening as the endpoint was $100 \%$. The average Harris hip score improved from $53.9( \pm 14.9)$ points preoperatively to $92.4( \pm 9.4)$ points postoperatively. The average postoperative pain score was 41.1 ( \pm 5.8$)$, and the average function scores were Gait $9.9( \pm 2.1)$, Walk $9.8( \pm 2.3)$, Stairs 2.9 ( \pm 2.1 ), and Range of Motion $4.7( \pm 0.3)$ points. There were 2 infections, one dislocation and one inner polyethylene liner fracture.

One patient, a 40-year-old woman, had a polyethylene liner fracture 11 months after the initial surgery. The implanted cup was stable and firm but the inner locking was damaged. She was diagnosed by a secondary osteoarthritis after traumatic hip injury. She was a rather high risk patient as she has some other comorbidity such as reflex sympathetic dystrophy.

The authors used solid cups without screws for 363 hips, 1 screw in 57 hips, 2 screws in 43 hips, 3 screws in 11 hips, and 4 screws in 2 hips. There were 20 auto-bone grafts and 1 allo-bone graft.

\section{Discussion}

Cementless acetabular cups for primary THA have had excellent outcomes, with failure rates typically less than 5\% for short- to mid-term follow-up [10]-[14]. In this study, the short-term survivorship of a new porous titanium cup demonstrated an excellent result in primary THA for the treatment of various hip arthritis etiologies. Regenerex Ring Loc+ cup showed 100\% survival with no aseptic loosening of the component. The clinical outcomes including Harris hip score were comparable to those reported previously for other highly porous metal components (Table 1 ).

The second generation porous metal acetabular components are roughly divided into 2 materials; tantalum and titanium. Porous Tantalum such as Trabecular Metal (Zimmer Biomet, Warsaw, IN) was introduced since late-1990s and both short-term and mid-term outcomes with this prosthesis were excellent [10]-[12]. On the other hand, porous titanium or porous coating titanium such as Gription (Deputy, Warsaw, IN), Tritanium (Stryker, Mahwah, NJ) and Stiktite (Smith and Nephew, Memphis, TN) as well as the current prosthesis Regenerex have been used since mid-2000s [13] [14]. Those prostheses have rougher porosity to get the initial stability. They also possess higher pore size ranging 100 to $600 \mu \mathrm{m}$ to induce cell migration and bone ingrowth. Gruen et al. demonstrated that the outcomes of 414 porous tantalum cups (Trabecular Metal) which had a 100\% survivorship at 2-year follow-up [10]. Macheras et al. experienced 151 hips with porous tantalum monoblock 
Table 1. Results with various 3-D porous metal cups.

\begin{tabular}{|c|c|c|c|c|c|c|c|c|}
\hline Author & $\begin{array}{l}\text { No. of Patients } \\
\text { (Hips) }\end{array}$ & $\begin{array}{l}\text { Approx. follow-up } \\
\text { (years) [range] }\end{array}$ & Metal type & Implant & $\begin{array}{l}\text { Preop } \\
\text { Harris }\end{array}$ & $\begin{array}{l}\text { stop } \\
\text { score }\end{array}$ & $\begin{array}{l}\text { Survivorship } \\
\text { (\%) }\end{array}$ & Notes \\
\hline Gruen [10] & 414 & $3[2-5]$ & Tantalum & Trabecular Metal & 49 & 90 & 100 & HHS of $82 \%$ of patients \\
\hline Macheras [11] & $138(151)$ & $9[8-10]$ & Tantalum & Trabecular Metal & 44 & 97 & 99 & $4.5 \%$ complication rate \\
\hline Malizos [12] & $218(240)$ & $5[3-9.5]$ & Tantalum & Trabecular Metal & 48 & 94 & 98.75 & \\
\hline Naudie [14] & 33 (33) & 2 & Titanium & Stiktite & & & 100 & Radiographic analysis \\
\hline Naziri [13] & $252(288)$ & $3[2-5]$ & Titanium & Tritanium & 53 & 91 & 100 & 1 reoperation for abductor repair \\
\hline Current study & $434(476)$ & $4.1[2.6-6.5]$ & Titanium & Regenerex & 53 & 92 & 100 & \\
\hline
\end{tabular}

acetabular component at 8- to 10-year follow-up and no component was revised or needed revision [11]. Naziri et al. reported 100\% survivorship of 288 THAs with a 3-D porous titanium cup (Tritanium) at up to 5-year follow-up [13].

The advantage of porous materials is their ability not only to provide a primary stability due to the scratch fit, but also to allow long-term implant stability related to bone ingrowth (osteoinductivity). The Regenerex shell is $16 \%$ rougher than other highly porous metal cups [7]. Small et al. demonstrated that Regenerex made $23 \%$ to $65 \%$ improvement in initial stability compared to plasma-sprayed cups [6]. But in a randomized trial with another cup (Stiktite), Naudie et al. reported that both a sintered bead porous surface and a 3-D porous titanium surface had great stability studying X-rays, and that there was no significant difference between the two after 2 years postoperatively [14]. Longer follow-up would show the difference due to the improved bone ingrowth and secondary stability.

Every new implant for joint arthroplasty needs long-term follow-up. Although a highly porous ingrowth surface, which increases the surface area of potential bone contact and improves cup stability, look like ideal conditions initially, Kleinhans et al. experienced significant third-body wear using a CoCrMo porous metal acetabular cup 8 years postoperatively [15]. No polyethylene wear or third-body was seen so far, and there was no damage report about porous tantalum or titanium acetabular cup.

In this study, there was one revision case for a polyethylene liner fracture and damage to Ring Loc+ locking system. A cementless modular acetabular titanium cup with a ring clamp was introduced as Ring Loc cup since the mid-1990s, and Olof et al. reported excellent outcomes with this cup and its liner as 98\% survival with 15 years follow-up [16]. Isaac et al. experienced 3 liner fractures out of 58 THAs with this Ring Loc cup up to 13 years follow-up [17]. In the current Regenerex cup, Ring Loc was improved with a more secure lock/unlock system, an extended rim and a larger apical hole to check the liner fit. This acetabular system does include a polyethylene liner and it may still retain some aspects leading to a liner fracture, though the incidence is not high.

There are several limitations in this study. First, it was not a prospective study and there is no control or matching group, so the authors can only compare the current outcomes to the past reports. Secondly, the indication of the surgery was not clear. 5 surgeons in the authors' institute used this prosthesis for primary THA, and the authors used it for both young active patients and elderly osteoporotic patients. The variability of number of screws used in the cup or bone grafts represents the wide spectrum of acetabular deformity. Although the follow-up is not long enough to judge the superiority of this prosthesis, the results are promising and longer follow-up studies will make a valuation.

\section{Conclusion}

Early clinical outcomes were successful with Regenerex Ring Loc+, a 3-D porous titanium acetabular cup. The survivorship with loosening of the cup as the endpoint was $100 \%$ during the follow-up time. There were one infection and one polyethylene liner fracture resulting in the revision surgery. Although further follow-up is necessary, the results are encouraging.

\section{References}

[1] Huo, M.H. (2002) What's New in Hip Arthroplasty. Journal of Bone and Joint Surgery (American), 84, $1894-1905$.

[2] Della Valle, C.J., Mesko, N.W., Quigley, L., Rosenberg, A.G., Jacobs, J.J. and Galante, J.O. (2009) Primary Total Hip 
Arthroplasty with a Porous-Coated Acetabular Component. A Concise Follow-Up, at a Minimum of Twenty Years, of Previous Reports. Journal of Bone and Joint Surgery (American), 91, 1130-1135. http://dx.doi.org/10.2106/JBJS.H.00168

[3] Engh, C.A., Hopper Jr., R.H. and Engh Jr., C.A. (2004) Long-Term Porous-Coated Cup Survivorship Using Spikes, Screws, and Press-Fitting for Initial Fixation. Journal of Arthroplasty, 19, 54-60. http://dx.doi.org/10.1016/j.arth.2004.06.004

[4] Herberts, P., Ahnfelt, L., Malchau, H., Strömberg, C. and Andersson, G.B. (1989) Multicenter Clinical Trials and Their Value in Assessing Total Joint Arthroplasty. Clinical Orthopedics Related Research, 249, 48-55. http://dx.doi.org/10.1097/00003086-198912000-00007

[5] Friedman, R.J., Black, J., Galante, J.O., Jacobs, J.J. and Skinner, H.B. (1994) Current Concepts in Orthopedic Biomaterials and Implant Fixation. Instructional Course Lecture, 43, 233-255.

[6] Small, S.R., Berend, M.E., Howard, L.A., Rogge, R.D., Buckley, C.A. and Ritter, M.A. (2013) High Initial Stability in Porous Titanium Acetabular Cups: A Biomechanical Study. Journal of Arthroplasty, 28, 510-516. http://dx.doi.org/10.1016/j.arth.2012.07.035

[7] Data on File at Zimmer Biomet, Inc. http://www.biomet.com/wps/wcm/connect/internet/4db3e9df-aa9a-4a9a-8b6b-ecef38094382/BOI0052.0_083107.pdf? MOD=AJPERES\&CONVERT $\mathrm{TO}=$ url\&CACHEID

[8] Bonicoli, E., Piolanti, N., Andreani, L., Parchi, P. and Lisanti, M. (2013) Preliminary Report with the Regenerex ${ }^{\mathrm{TM}}$ Revision Shell: Clinical, Functional, and Radiographic Evaluation with a Mean Follow-Up of 25 Months. European Orthopedic Traumatology, 4, 9-14. http://dx.doi.org/10.1007/s12570-012-0146-2

[9] Sillesen, N.H., Greene, M.E., Nebergall, A.K., Nielsen, P.T., Laursen, M.B., Troelsen, A. and Malchau, H. (2015) Three Year RSA Evaluation of Vitamin E Diffused Highly Cross-linked Polyethylene Liners and Cup Stability. Journal of Arthroplasty, 30, 1260-1264. http://dx.doi.org/10.1016/j.arth.2015.02.018

[10] Gruen, T.A., Poggie, R.A., Lewallen, D.G., Hanssen, A.D., Lewis, R.J., O’Keefe, T.J., Stulberg, S.D. and Sutherland, C.J. (2005) Radiographic Evaluation of a Monoblock Acetabular Component: A Multicenter Study with 2- to 5-Year Results. Journal of Arthroplasty, 20, 369-378. http://dx.doi.org/10.1016/j.arth.2004.12.049

[11] Macheras, G., Kateros, K., Kostakos, A., Koutsostathis, S., Danomaras, D. and Papagelopoulos, P.J. (2009) Eight- to Ten-Year Clinical and Radiographic Outcome of a Porous Tantalum Monoblock Acetabular Component. Journal of Arthroplasty, 24, 705-709. http://dx.doi.org/10.1016/j.arth.2008.06.020

[12] Malizos, K.N., Bargiotas, K., Papatheodorou, L., Hantes, M. and Karachalios T. (2008) Survivorship of Monoblock Trabecular Metal Cups in Primary THA: Midterm Results. Clinical Orthopedics Related Research, 466, 159-166. http://dx.doi.org/10.1007/s11999-007-0008-3

[13] Naziri, Q., Issa, K., Pivec, R., Harwin, S.F., Delanois, R.E. and Mont, M.A. (2013) Excellent Results of Primary THA Using a Highly Porous Titanium Cup. ORTHOPEDICS, 36, e390-e394. http://dx.doi.org/10.3928/01477447-20130327-10

[14] Naudie, D.D.R., Somerville, L., Korczak, A., Yuan, X., McCalden, R.W., Holdsworth, D. and Bourne, R.B. (2013) A Randomized Trial Comparing Acetabular Component Fixation of Two Porous Ingrowth Surfaces Using RSA. Journal of Arthroplasty, 28, 28-52. http://dx.doi.org/10.1016/j.arth.2013.06.041

[15] Kleinhans, J.A., Jakubowitz, E., Seeger, J.B., Heisel, C. and Kretzer, J.P. (2009) Macroscopic Third-Body Wear Caused by Porous Metal Surface Fragments in Total Hip Arthroplasty. ORTHOPEDICS, 32, 364. http://dx.doi.org/10.3928/01477447-20090501-06

[16] Olof, S., Mats, S., Olle, M., Ake, J., Torbjörn, A. and Henrik, B. (2009) The Ring Loc Liner Compared with the Hexloc Liner in Total Hip Arthroplasty. Orthopedic Reviews, 1, e16.

[17] Isaac, D.L., Forder, J., Skyrme, A.D. and James, S.E. (2007) The Biomet Bi-Metric Total Hip Arthroplasty and Universal Acetabular Cup: High Polyethylene Failure Rate in the Medium Term. Journal of Arthroplasty, 22, 697-700. http://dx.doi.org/10.1016/j.arth.2006.09.015 